\title{
Tofacitinib in the treatment of active rheumatoid arthritis - single-centre experience
}

\author{
Marta Madej ${ }^{1,2}$, Patryk Woytala ${ }^{1}$, Marek Frankowski ${ }^{1,2}$, tukasz Lubiński ${ }^{1}$, Renata Sokolik ${ }^{1,2}$, \\ Agata Sebastian $^{1,2}$, Beata Maciążek-Chyra ${ }^{1}$, Piotr Wiland ${ }^{1,2}$ \\ ${ }^{1}$ Clinic of Rheumatology and Internal Medicine, Wroclaw University Hospital, Poland \\ ${ }^{2}$ Department and Clinic of Rheumatology and Internal Medicine, Wroclaw Medical University, Poland
}

\begin{abstract}
Objectives: To assess the efficacy and safety profile of tofacitinib taken orally at a dose of $10 \mathrm{mg} /$ day in patients with severe active rheumatoid arthritis (RA).

Material and methods: The retrospective observational study included 10 patients ( 6 women and 4 men) with RA treated with tofacitinib. All the patients had high disease activity (DAS28 [ESR] > 5.1), despite therapy with two synthetic disease-modifying antirheumatic drugs (DMARDs). Before the initiation of treatment, routine laboratory tests were performed, and disease activity was assessed in all the subjects.

Results: The mean age of the patients in the study group was 58.18 years (43-67). The average duration of RA was 9.9 years (2-24). The mean baseline value of DAS28 (ESR) was 6.37. Tofacitinib was used in combination with a conventional DMARD in all study subjects: with methotrexate in the majority of patients, and with leflunomide and an antimalarial drug in three patients. The mean duration of therapy with tofacitinib was 7.57 months (3.9-10.8). A significant decrease in the disease activity was observed $(p<0.05)$. A reduction in DAS28 (ESR) score was seen already after the first month of therapy, and the trend was maintained during subsequent months of follow-up. The mean value of DAS28 (ESR) after 6 months was 2.78. A slight increase in the serum concentration of HDL cholesterol was observed during treatment. In one patient symptoms of chronic upper respiratory tract infection led to discontinuation of the drug. The observed adverse events were of mild/ moderate degree.

Conclusions: The results of our retrospective observational study conducted in the setting of daily clinical practice confirm a good clinical response to tofacitinib. Despite the observed adverse effects, in the light of the available data tofacitinib demonstrates a favourable safety profile. JAK kinase inhibitors - a new class of drugs - will enable a wider population of patients to achieve remission or low disease activity.
\end{abstract}

Key words: rheumatoid arthritis, disease activity, tofacitinib, disease-modifying antirheumatic drugs.

\section{Introduction}

Rheumatoid arthritis (RA) is treated primarily by pharmacotherapy, which aims to induce clinical remission or at least achieve low disease activity. Synthetic disease-modifying antirheumatic drugs (DMARDs) are a group of medicines which can be divided into two sub- groups: conventional and targeted [1]. Targeted DMARDs comprise JAK kinase inhibitors including tofacitinib. The drug inhibits the activity of Janus kinases JAK1 and JAK3 and to a lesser extent also JAK2 and TYK2 [2]. Janus kinases are a group of enzymes which play a crucial role in the process of transduction of receptor signals into

Address for correspondence:

Marta Madej, Clinic of Rheumatology and Internal Medicine, Wroclaw University Hospital, 213 Borowska St., 50-556 Wrocław, Poland, e-mail: marta.madej@poczta.fm

Submitted: 30.05.2019; Accepted: 16.07.2019 
cells (for proinflammatory cytokines, among others), which ultimately activates the inflammatory process. The inhibition of JAK kinases by tofacitinib attenuates signal transduction from the receptors for interferon types I and II, and interleukins (IL-2, IL-4, IL-6, IL-7, IL-9, IL-15, IL-21), leading to modification of the inflammatory response [3].

According to the approvals granted by the European Medicines Agency (EMA) and the U.S. Food and Drug Administration (FDA), tofacitinib has indications in the treatment of moderate to severe rheumatoid arthritis in adult patients with an inadequate response or intolerance to at least one DMARD. The drug can be used in combination with methotrexate or in monotherapy [3].

The available data on the efficacy and safety of tofacitinib treatment are based on phase 2 and 3 clinical studies, their extension phases, and register-based observational studies. In Poland, experience with the use of tofacitinib is limited, which is mainly due to restricted access to the drug attributed to economic factors. To our knowledge, the presented findings are the first Polish report addressing the issue of efficacy and safety of tofacitinib use in the setting of daily clinical practice.

The aim of the study was to assess the efficacy of treatment with tofacitinib used orally at a dose of $5 \mathrm{mg}$ twice a day in patients with severe active RA. The safety profile and drug tolerance were also evaluated.

\section{Material and methods}

Our retrospective observational study included a total of 10 patients ( 6 women and 4 men) with rheumatoid arthritis, hospitalized in the Clinic of Rheumatology and Internal Medicine, Wroclaw University Hospital, between June 2018 and April 2019, and treated with tofacitinib. The study subjects were $>18$ years of age, diagnosed with rheumatoid arthritis on the basis of the 2010 ACR/EULAR (American College of Rheumatology/European League Against Rheumatism) criteria or the 1987 ACR criteria. All the patients had high disease activity as measured by the Disease Activity Score 28 (DAS28) of > 5.1 despite treatment with at least two synthetic DMARDs for a minimum of 4 months with each of the drugs. In view of the lack of response to prior therapy tofacitinib at a dose of $5 \mathrm{mg}$ twice a day was added to the therapeutic regimen.

Before starting treatment with tofacitinib all the patients underwent routine examinations performed to assess eligibility for biologic treatment within the framework of the National Health Fund drug programmes, including acute phase markers (ESR, CRP), peripheral blood analysis with differential, liver aminotransferase activity, full lipid profile (total cholesterol,
HDL, LDL, triglycerides), kidney function parameters, rheumatoid factor (RF) and anti-citrullinated protein antibodies (ACPA), tests to exclude infection with HBV and HCV, and HIV (HBsAg, anti-HBC antibodies, anti-HCV antibodies, anti-HIV antibodies), the QuantiFERON-TB Gold blood test, and radiological chest examination. Patients with contraindications included in the summary of product characteristics for tofacitinib were excluded from treatment.

The activity of the disease was evaluated in every patient using the DAS28 score based on the ESR value.

Evaluation of disease activity and routine examinations were performed in every subject at baseline, and afterwards after 1, 3, 6 and 9 months of tofacitinib therapy.

All the patients were informed about the benefits and potential adverse effects associated with the use of tofacitinib, and gave their written consent to the treatment. Since the study had a retrospective design (based on an analysis of medical records), the consent of the bioethics committee was not required. The drug was used according to its approved indications.

\section{Statistical analysis}

The statistical analysis was conducted using the Statistica package (version 10.0). The significance level was set at $5 \%$. The normality of distributions was verified with the Kolmogorov-Smirnov test, and the significance of changes in parameter values over time was determined using the ANOVA method for repeated measurements. The pairs of observations between which statistically significant differences occur (in individual measurements) were identified using the least significant difference (LSD) test.

\section{Results}

The demographic and clinical characteristics of the study group are presented in Table I.

Tofacitinib was used in combination with a conventional DMARD in each patient in the study group. Eight patients were treated with tofacitinib in combination with methotrexate (mean dose $18.75 \mathrm{mg}$ per week (range 10-25 mg per week), 5/8 subcutaneously, 3/8 orally). In one patient, two synthetic DMARDs (methotrexate and leflunomide) were used concurrently at the time of introducing tofacitinib because of high disease activity. In this case, leflunomide was discontinued after three months in view of the good response to treatment, and combined therapy with methotrexate and tofacitinib was continued. Methotrexate was not used in two drug-intolerant patients. Instead, these patients were treated with other conventional DMARDs - one with leflunomide, and one with hydroxychloroquine. 
Table I. Clinical characteristics of study group

\begin{tabular}{|lc|}
\hline Clinical characteristics & Patients \\
\hline Number of participants & 10 \\
\hline Gender & $\begin{array}{c}\text { Male 4/10 } \\
\text { Female 6/10 }\end{array}$ \\
\hline Age (years) & $57.4(43-67)$ \\
\hline Duration of RA (years) & $9.90(2-24)$ \\
\hline $\begin{array}{l}\text { Duration of tofacitinib treatment } \\
\text { (months) }\end{array}$ & $7.57(3.9-10.8)$ \\
\hline Rheumatoid factor positive & $10 / 10$ \\
\hline ACPA positive & $10 / 10$ \\
\hline Methotrexate treatment & $8 / 10$ \\
\hline Previous methotrexate treatment & $10 / 10$ \\
\hline Previous sulfasalazine treatment & $7 / 10$ \\
\hline Previous leflunomide treatment & $6 / 10$ \\
\hline $\begin{array}{l}\text { Previous hydroxychloroquine/chloroquine } \\
\text { treatment }\end{array}$ & $5 / 10$ \\
\hline Previous ciclosporin treatment & $2 / 10$ \\
\hline
\end{tabular}

Table II. Results of ANOVA test for selected laboratory parameters

\begin{tabular}{|ll|}
\hline Parameter & $p$-value \\
\hline DAS28 (ESR) & 0.0000 \\
\hline Total cholesterol & 0.0794 \\
\hline LDL cholesterol & 0.2945 \\
\hline HDL cholesterol & 0.0151 \\
\hline Triglycerides & 0.9010 \\
\hline Lymphocytes & 0.3896 \\
\hline Neutrophils & 0.6126 \\
\hline Haemoglobin & 0.2568 \\
\hline
\end{tabular}

Table III. Basic parameter statistics for which statistically significant changes in mean values were observed in successive measurements

\begin{tabular}{|lccccc|}
\hline Parameter & Mean & Median & Min & Max & SD \\
\hline DAS28 - baseline & 6.37 & 6.34 & 5.08 & 8.26 & 1.04 \\
\hline DAS28 - month 1 & 4.59 & 4.54 & 1.61 & 7.90 & 1.95 \\
\hline DAS28 - month 3 & 3.38 & 3.24 & 1.74 & 6.69 & 1.38 \\
\hline DAS28 - month 6 & 2.78 & 1.90 & 1.54 & 5.09 & 1.49 \\
\hline $\begin{array}{l}\text { Cholesterol HDL } \\
\text { (mg/dl) - baseline }\end{array}$ & 56 & 57 & 38 & 70 & 12 \\
\hline $\begin{array}{l}\text { Cholesterol HDL } \\
\text { (mg/dl) - month 1 }\end{array}$ & 66 & 66 & 46 & 88 & 15 \\
\hline $\begin{array}{l}\text { Cholesterol HDL } \\
\text { (mg/dl) - month 3 }\end{array}$ & 67 & 65 & 40 & 93 & 17 \\
\hline $\begin{array}{l}\text { Cholesterol HDL } \\
\text { (mg/dl) - month 6 }\end{array}$ & 67 & 64 & 45 & 93 & 17 \\
\hline
\end{tabular}

Two patients had a history of biologic drug treatment. One patient received rituximab within the framework of a clinical study. The treatment was completed according to the study protocol. At the time of starting tofacitinib therapy, over five years had passed since the last administration of rituximab. The other patient was treated with adalimumab, infliximab and tocilizumab, but biologic therapy with all these drugs was found to be unsuccessful. Treatment with the last of the drugs listed above - tocilizumab - was completed two years before the initiation of tofacitinib.

Currently, a total of 8 patients continue tofacitinib therapy. In one subject, therapy was prematurely terminated because of recurrent infections of the upper respiratory tract. The patient presented with symptoms of mild and moderate severity, not requiring hospitalization, which occurred approximately 4 months after the initiation of tofacitinib therapy. The patient was treated with oral antibiotics (amoxicillin 7 days, cefuroxime 5 days, clarithromycin 7 days), with slight improvement. However, the symptoms resolved after the discontinuation of tofacitinib. In another patient, primary inefficacy of tofacitinib therapy was determined. The treatment was terminated after 3 months with persistently high disease activity.

At the time of starting tofacitinib therapy, the dose of glucocorticosteroids (expressed as prednisone-equivalent) in the study group did not exceed $10 \mathrm{mg} /$ day. The dose of glucocorticosteroids was reduced in all patients who had an adequate response to treatment.

The activity of the disease and the studied laboratory parameters were evaluated in all patients after 1 and 3 months of therapy, in 7 subjects after 6 months, and in 3 subjects after 9 months of treatment.

Table II presents the results of the ANOVA test verifying whether the mean values of the study parameters differ from one other in successive measurements over time in a statistically significant manner. As the study group was small, the statistical analysis excluded measurements performed at month 9 (the study was conducted in real-time conditions, and at the time of clinical data analysis after 9 months of therapy there were only 3 patients left).

The analysis demonstrated a statistically significant difference in consecutive DAS28 and HDL cholesterol measurements. There were no significant differences in the measured values of total cholesterol, LDL cholesterol, triglycerides, lymphocytes, neutrophils or haemoglobin. Table III presents the basic statistics for the parameters where the mean values changed significantly in successive measurements.

The analysis showed a significant decrease in the DAS28 (ESR) value $(p<0.05)$. The mean baseline score 
in the study group was 6.37. A statistically significant decrease in disease activity was observed already in the first assessment performed after 4 weeks of tofacitinib therapy (DAS28 [ESR] 6.37 vs. 4.59, $p<0.05$ ). Further reductions in disease activity were noted in subsequent months. The mean DAS28 (ESR) value in the assessment performed after 6 months was 2.78 (Fig. 1).

Table IV presents the quantitative breakdown of patients depending on the DAS28 value.

The analyses revealed no statistically significant differences among the mean values of total cholesterol and LDL obtained in successive measurements ( $p=0.07938$ and $p=0.2945$, respectively). However, statistically significant differences were observed among mean $\mathrm{HDL}$ values in successive measurements ( $p=0.0151)$. The mean concentration of HDL in the study group at baseline was $56 \mathrm{mg} / \mathrm{dl}$. After the first month of treatment, a significant increase in HDL cholesterol concentrations was observed, reaching the mean value of $66 \mathrm{mg} / \mathrm{dl}$ ( $p=0.0039$ ). However, in further follow-up, the mean values of HDL cholesterol concentrations remained at a constant level (Fig. 2). The results of the LSD test failed to demonstrate any statistically significant differences in the mean values of HDL cholesterol evaluated after 1 , 3 , and 6 months of treatment.

Furthermore, there were no statistically significant changes in liver enzyme activity levels or blood serum creatinine concentrations during tofacitinib therapy (statistics not included in the paper).

In the course of tofacitinib treatment, one patient underwent arthroplasty of the right metacarpophalangeal joints II to V. Tofacitinib therapy was suspended for the duration of the procedure. The perioperative and postoperative course was uncomplicated.

\section{Discussion}

Based on the current EULAR recommendations (update 2016) tofacitinib can be used as second-line treatment in patients with poor prognostic factors (high level of serological markers of RA, high disease activity, early joint destruction) after unsuccessful therapy with a conventional synthetic DMARD, or as third-line treatment after failing therapy with a biologic drug [4].

The findings of our observational study conducted in the setting of real-life clinical practice confirm that tofacitinib can be an effective therapeutic option in the patient groups described above. The analysis performed after 90 days of therapy showed remission/low disease activity (DAS28 $\leq 3.2$ ) in 5/10 patients, and moderate disease activity $(5.1 \geq$ DAS28 $>3.2)$ in another four patients.
Table IV. Disease activity after 1 and 3 months of therapy

\begin{tabular}{|lccc|}
\hline Time & \multicolumn{3}{c|}{ Disease activity based on DAS28 (ESR) } \\
\cline { 2 - 4 } & Low/remission & Moderate & High \\
\hline $\begin{array}{l}\text { After 1 month } \\
\text { ( } n \text { of patients) }\end{array}$ & $3 / 10$ & $3 / 10$ & $4 / 10$ \\
\hline $\begin{array}{l}\text { After 3 months } \\
\text { ( } n \text { of patients) }\end{array}$ & $5 / 10$ & $4 / 10$ & $1 / 10$ \\
\hline
\end{tabular}

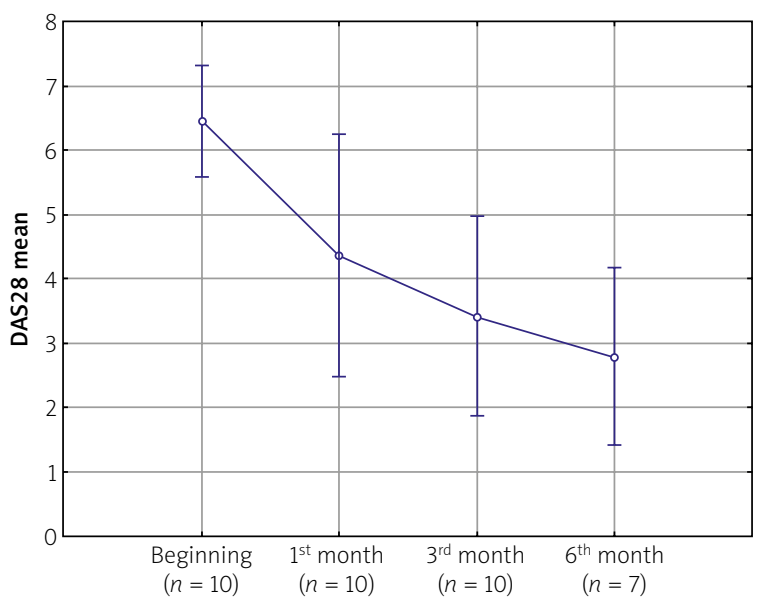

Fig. 1. Mean DAS28 score (95\% confidence interval).

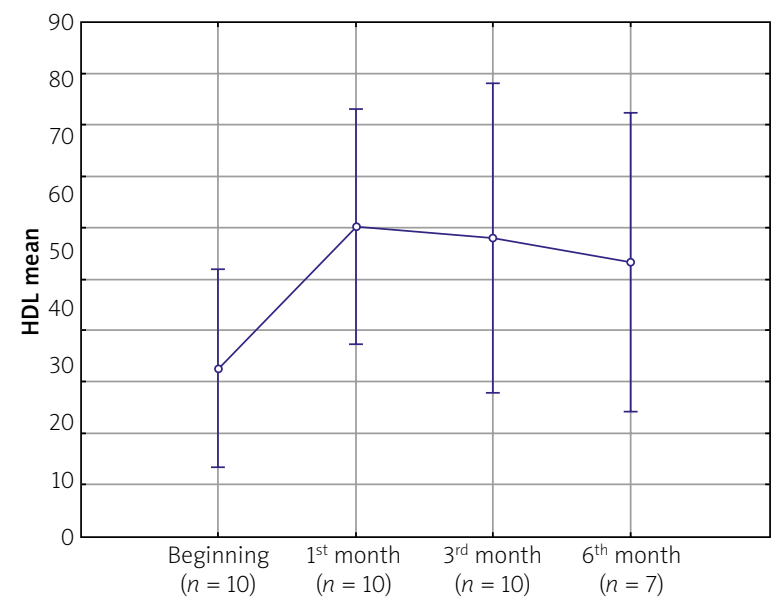

Fig. 2. Mean HDL cholesterol (95\% confidence interval).

It needs to be noted that all the patients included in our analysis had unfavourable prognostic factors (lack of response to at least two synthetic DMARDs, high titres of RF and ACPA antibodies, high disease activity).

According to literature data tofacitinib has a beneficial effect on disease activity, improves patient functioning, and slows down the progression of radiological 
changes. In a phase 3 clinical study (ORAL Solo) comparing tofacitinib (at doses of $5 \mathrm{mg} /$ day and $10 \mathrm{mg} /$ day) with placebo in the population of RA patients, a significant and dose-dependent increase was noted in the percentage of patients who achieved an ACR20, ACR50 and ACR70 response after 3 months of therapy in the population treated with a JAK inhibitor [5].

Also, a significant improvement in the patients' functional status assessed by the HAQ-DI (Health Assessment Questionnaire - Disability Index) questionnaire was seen in the arm of tofacitinib-treated patients. An improvement in patient functioning was noted already after two weeks of therapy, and the trend continued throughout the follow-up period of 6 months. Interestingly, there were no significant differences in the frequency of clinical remission (assessed by DAS28 [ESR]) between the drug and placebo after 3 months of therapy.

Our study did not include an evaluation of the effect of tofacitinib on the radiological progression because of the short follow-up period and small number of study patients. Analyses based on randomized clinical studies show that the progression of radiological changes assessed by the modified Sharp/van der Heijde score in the group of patients treated with tofacitinib $(10 \mathrm{mg} /$ day and $20 \mathrm{mg} /$ day) was significantly lower after 6 months of treatment than among patients using methotrexate (MTX) in monotherapy [6].

The beneficial effect of the drug with regard to radiological progression was consistent with the clinical response assessed by the ACR70 score. A 12-month observational study (ORAL Scan) confirmed the favourable effect of the drug on inhibiting the progression of radiological changes in the course of the disease [7]. In the $12^{\text {th }}$ month, a statistically significant change in the Sharp/van der Heijde score was observed for the tofacitinib dose of $20 \mathrm{mg} /$ day. Also, a statistically significant reduction in the progression of joint space narrowing was noted in the assessment performed after 12 months of treatment for both doses of the drug (10 mg/day and $20 \mathrm{mg} /$ day).

In our study group, there was only one patient treated with tofacitinib after previous unsuccessful biologic treatment. Notably, despite the initial good response after 3 months of treatment (DAS28 [ESR] -3.42), the patient experienced exacerbation of the disease (DAS28 [ESR] > 5.1) demonstrated in the assessment after 180 days of therapy. The literature reports confirm that the use of a JAK kinase inhibitor can produce a beneficial therapeutic effect also in this population of patients. The percentage of study subjects who, after unsuccessful therapy with a TNF- $\alpha$ inhibitor, achieved remission during combination therapy with tofacitinib and methotrexate, was correlated with the dose of JAK kinase in- hibitor (10 mg/day or $20 \mathrm{mg} /$ day), and was $6.7 \%$ and $8.8 \%$, respectively [8].

All the patients enrolled in our study used tofacitinib in combination with MTX, except for two subjects who received combination treatment with tofacitinib and an antimalarial drug or leflunomide because of MTX intolerance. Furthermore, one patient was treated with tofacitinib in combination with MTX and leflunomide because of high disease activity at baseline. Tofacitinib can be used alone or in combination with MTX, as specified in the summary of product characteristics. However, as daily clinical practice shows, in specific cases it may be necessary to use a JAK inhibitor with a synthetic DMARD other than MTX. The currently available data on this topic are limited. In a study by Kremer et al. (ORAL Sync), 62.5-73.4\% of patients used a synthetic DMARD - in most cases (79\%) MTX - in combination with tofacitinib. However, as many as $25.3-37.5 \%$ of subjects used at least two synthetic DMARDs (including non-MTX DMARDs) concurrently with the JAK kinase inhibitor [9].

As there are no large randomized clinical trials comparing combination therapy with MTX/tofacitinib and synthetic DMARD (nonMTX)/tofacitinib, particularly with respect to safety and the risk of adverse effects, this therapeutic regimen requires more in-depth study. In the light of the findings of the ORAL Sync study, and the statement of the expert group of the Polish Society of Rheumatology, tofacitinib can be used in combination with either methotrexate or another synthetic DMARD $[9,10]$.

Treatment with tofacitinib has been found to be associated with laboratory test abnormalities including neutropenia, lymphopenia, thrombocytopenia, reduced haemoglobin concentrations, and elevated levels of transaminases, creatinine and serum lipids. The mechanism of these phenomena is not fully understood. The results of patient follow-up spanning more than 12 months indicate that an increase in creatinine concentrations most commonly occurs during the first three months of treatment. A pooled analysis of five phase 3 studies $(n=3,315)$ and two long-term extension studies $(n=3,227)$ demonstrated an increase in serum creatinine concentration by $\geq 33 \%$ over the baseline in the period from the onset to 3 months after the initiation of therapy, in $1.4 \%$ and $1.9 \%$ of patients using a dose of $10 \mathrm{mg} /$ day and $20 \mathrm{mg} /$ day, respectively [11].

In most cases, serum creatinine concentrations plateaued over the course of treatment and remained within the normal range. The highest increase in serum creatinine concentrations was noted in patients with high baseline CRP values and a significant decrease in CRP levels occurring in response to tofacitinib treatment. In addition, isolated cases of acute renal failure $(n=22)$ 
were reported during tofacitinib therapy, but in all cases the disorder coexisted with another severe condition.

In the present analysis, a statistically significant increase in HDL cholesterol concentration was noted already after a month of tofacitinib therapy. However, the abnormalities were clinically insignificant, and did not require the discontinuation of treatment. What is more, patient follow-up in subsequent months demonstrated that the HDL cholesterol levels had stabilized, with a slight falling trend seen in HDL serum concentrations. We did not observe a clinically significant increase in the concentration of creatinine and liver enzymes in the analysed group.

The increase in the HDL cholesterol concentration in blood serum is consistent with the literature data. A pooled analysis of phase 2 and 3 clinical trials evaluating the effect of tofacitinib on the lipid profile shows that the drug is associated with an increase in HDL and LDL cholesterol concentrations by about $10-20 \%$ of baseline values [12]. In the majority of cases, changes in lipid concentrations are observed in the early phase of treatment (during the first month) and normalize after 3 months of tofacitinib therapy. One of the studies showed a beneficial effect of adding a low dose of statin to tofacitinib therapy, which was associated with a decrease in total cholesterol, LDL and triglyceride levels below baseline values [13].

An analysis of the risk of haematological complications associated with tofacitinib therapy shows that they usually present as mild disorders and in most cases do not require the treatment to be stopped [14].

Tofacitinib was discontinued in one patient in our study group following the development of adverse effects during the therapy. The observed effects, however, did not warrant the classification as serious adverse events. A pooled analysis of the risk of adverse effects associated with tofacitinib (data cut-off: March 2015, drug exposure over 8 years) summarizing data from phase 1-3 clinical studies and long-term extension studies showed no significant differences compared to previously reported adverse effects of the drug [15].

Data analysis demonstrated that long-term tofacitinib treatment had not contributed to an increase in the risk of adverse effects, and their frequency and nature were similar to those reported in phase 3 trials. The most widespread adverse effects were infections: pneumonia, shingles, urinary tract infections, cellulitis, and symptoms of gastrointestinal intolerance. Post-marketing data indicate that the most common serious adverse events include infections of various sites, with reporting rates (RR) of 2.57/100 patient-years, and the highest incidence noted during the first year of treatment [16]. The incidence of cancer was shown to be 0.45 per 100 patient-years, and the most frequently occurring cancer types included non-melanoma skin carcinomas. Regarding the epidemiology of tuberculosis, the risk of the disease in tofacitinib-treated patients was similar to that in patients receiving biologic drugs [16].

In our study population, there were no cases of Herpes zoster, which may be attributed to the relatively short period of patient follow-up and drug exposure. None of the patients had a history of disseminated Varicella zoster infection and/or shingles. Based on the available data, the risk of the complication in patients treated with tofacitinib is higher than in patients receiving therapy with TNF- $\alpha$ inhibitors or other JAK kinase inhibitors. In most cases (94\%) shingles was limited to a single dermatome and had a mild course $[15,17]$. The risk of reactivation of latent infection depends on age, and it is the highest in patients receiving tofacitinib at a dose of $20 \mathrm{mg} /$ day, treated with glucocorticosteroids, and of Asian origin [17]

The incidence rate (IR) in the Eastern European population is estimated at 2.4, and among Asians at 6.1, with the highest incidence rate found in Japan (IR 8.0). In addition, an increased risk of infection reactivation occurs in patients with deep lymphopenia $(<1,000$ cells $/ \mathrm{ml}$ ) and previously treated with more than two biologic drugs [3]. Only in $8 \%$ of cases did the development of Herpes zoster necessitate permanent discontinuation of the drug. The likely link between tofacitinib therapy and the reactivation of infection is attributable to the effect of the drug on interferon activity [17].

The main limitation of our study is the low number of patients due to the restricted availability of the drug in the Polish healthcare system. A strength of the study is the presentation of our own experiences with using the drug with a novel mechanism of action in the setting of daily clinical practice.

\section{Conclusions}

Extensive data from clinical trials confirm the efficacy of tofacitinib used in monotherapy and in combination with a synthetic DMARD, both in the population of patients who have not been previously treated with DMARDs, and in patients with no response to either synthetic or biological DMARDs. The therapeutic effect of tofacitinib is comparable to the efficacy of biologic drugs including TNF- $\alpha$ inhibitors and drugs with a different mechanism of action $[18,19]$. Good clinical response to treatment was also demonstrated in our retrospective observational study conducted in the setting of daily clinical practice. Despite the identified adverse effects, in the light of the available data tofacitinib has a favourable safety profile. JAK kinase inhibitors, a new class of 
drugs expanding the range of therapeutic options, wil enable a wider population of patients to achieve the therapeutic goals of remission or low disease activity.

The authors declare no conflict of interest. The study has not received any specific financial support.

\section{References}

1. Smolen JS, van der Heijde D, Machold KP, et al. Proposal for a new nomenclature of disease-modifying antirheumatic drugs. Ann Rheum Dis 2014; 73: 3-5.

2. Hodge JA, Kawabata TT, Krishnaswami S, et al. The mechanism of action of tofacitinib - an oral Janus kinase inhibitor for the treatment of rheumatoid arthritis. Clin Exp Rheumatol 2016; 34: 318-328.

3. Tofacitinib: Summary of Product Characteristics. http://www. ema.europa.eu/docs/en_GB/document_library/EPAR_-_Product_Information/human/004214/WC500224911.pdf

4. Smolen JS, Landewé R, Bijlsma J, et al. EULAR recommendations for the management of rheumatoid arthritis with synthetic and biological disease-modifying antirheumatic drugs: 2016 update. Ann Rheum Dis 2017; 76: 960-977.

5. Fleischmann R, Kremer J, Cush J, et al. Placebo controlled trial of tofacitinib monotherapy in rheumatoid arthritis. N Engl J Med 2012; 367: 495-507.

6. Lee EB, Fleischmann R, Hall S, Wilkinson B, et al. Tofacitinib versus methotrexate in rheumatoid arthritis. $N$ Engl J Med 2014; 370: 2377-2386.

7. van der Heijde D, Tanaka Y, Fleischmann R, et al. Tofacitinib (CP-690,550) in patients with rheumatoid arthritis receiving methotrexate: twelve-month data from a twenty-four-month phase III randomized radiographic study. Arthritis Rheum 2013; 65: 559-570.

8. Burmester GR, Blanco R, Charles-Schoeman C, et al. Tofacitinib (CP-690,550) in combination with methotrexate in patients with active rheumatoid arthritis with an inadequate response to tumour necrosis factor inhibitors: a randomized phase 3 trial. Lancet 2013; 381: 451-460.
9. Kremer J, Li ZG, Hall S, et al. Tofacitinib in combination with nonbiologic disease-modifying antirheumatic drugs in patients with active rheumatoid arthritis: a randomized trial. Ann Intern Med 2013; 159: 253-261.

10. Kucharz EJ, Stajszczyk M, Kotulska-Kucharz A, et al. Tofacitinib in the treatment of patients with rheumatoid arthritis: position statement of experts of the Polish Society for Rheumatology. Reumatologia 2018; 56: 203-211.

11. Isaacs JD, Zuckerman A, Krishnaswami S, et al. Changes in serum creatinine in patients with active rheumatoid arthritis treated with tofacitinib: results from clinical trials. Arthritis Res Ther 2014; 16: R158.

12. Charles-Schoeman C, Gonzalez-Gay MA, Kaplan I, et al. Effects of tofacitinib and other DMARDs on lipid profiles in rheumatoid arthritis: implications for the rheumatologist. Semin Arthritis Rheum 2016; 46: 71-80.

13. McInnes IB, Kim HY, Lee SH, et al. Open-label tofacitinib and double-blind atorvastatin in rheumatoid arthritis patients: a randomised study. Ann Rheum Dis 2014; 73: 124-131.

14. Lundquist LM, Cole SW, Sikes ML. Efficacy and safety of tofacitinib for treatment of rheumatoid arthritis. World J Orthop 2014; 5: 504-511.

15. Cohen SB, Tanaka Y, Mariette $X$, et al. Long-term safety of tofacitinib for the treatment of rheumatoid arthritis up to 8.5 years: integrated analysis of data from the global clinical trials. Ann Rheum Dis 2017; 76: 1253-1262.

16. Cohen S, Curtis JR, DeMasi R, et al. Worldwide, 3-Year, Post-Marketing Surveillance Experience with Tofacitinib in Rheumatoid Arthritis. Rheumatol Ther 2018; 5: 283-291.

17. Winthrop KL, Curtis JR, Lindsey S, et al. Herpes Zoster and Tofacitinib: Clinical Outcomes and the Risk of Concomitant Therapy. Arthritis Rheumatol 2017; 69: 1960-1968.

18. Fleischmann R, Mysler E, Hall S, et al. Efficacy and safety of tofacitinib monotherapy, tofacitinib with methotrexate, and adalimumab with methotrexate in patients with rheumatoid arthritis (ORAL Strategy): a phase 3b/4, double-blind, head-to-head, randomised controlled trial. Lancet 2017; 390: 457-468.

19. Nakamura Y, Suzuki T, Yamazaki H, Kato H. Tofacitinib Versus Non-Tumor Necrosis Factor Biologics for Patients With Active Rheumatoid Arthritis. Arch Rheumatol 2017; 33: 154-159. 